\title{
Cultura e a consulta de medicina geral e familiar: um caso de síndroma de Dhat
}

Sara Antunes*

\section{RESUMO}

Introdução: A síndroma de Dhat consiste na preocupação em que a perda de sémen seja causadora de perda de energia vital e, consequentemente, de doença. Ocorre principalmente em homens jovens e sul-asiáticos e apresenta-se sob a forma de sintomas inespecíficos (como fraqueza, tristeza e ansiedade). Com este caso pretende-se discutir a importância do contexto sociocultural de um utente e a sua abordagem em cuidados de saúde primários.

Descrição do caso: Descreve-se o caso de um homem de 44 anos, natural do Bangladesh, sem antecedentes pessoais ou familiares relevantes. Membro de uma família nuclear em fase IV do ciclo de vida de Duvall no país de origem, emigrou para Portugal em 2010, onde vive sozinho. Sem seguimento anterior na USF, pediu consulta não programada por queixas de fraqueza e ansiedade com um mês de evolução e sensação de morte iminente desde há poucos dias. Associava os sintomas à emissão de um líquido branco durante a micção. Negou contactos sexuais nos últimos sete anos. Tendo em conta a hipótese diagnóstica de síndroma de Dhat realizou-se escuta ativa, com reconhecimento do sofrimento e tranquilização do utente. Foram marcadas consultas de reavaliação, às quais o utente aderiu e durante as quais a relação terapêutica médico-doente foi sendo estabelecida, com melhoria progressiva da sintomatologia referida.

Comentário: A multiculturalidade assume um papel cada vez mais relevante nas sociedades. O médico de família, como prestador de cuidados de primeira linha, pode ter um papel importante na identificação de síndromas culturais. O conhecimento desta síndroma em particular permitiu uma abordagem individualizada, evitando medidas terapêuticas desadequadas, com evolução favorável do caso.

Palavras-chave: Diversidade cultural; Síndroma de Dhat; Medicina geral e familiar.

\section{INTRODUÇÃO}

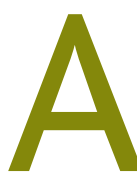
síndroma de Dhat tem sido descrita mais frequentemente em países do Sul da Ásia. Apresenta-se sob a forma de sintomas inespecíficos (como fraqueza, tristeza e ansiedade), que se iniciam após a emissão de fluído branco pelo pénis. ${ }^{1-7}$

Com este relato de caso pretende-se discutir a importância do contexto sociocultural de um utente, através da identificação de uma síndroma cultural (mais especificamente a síndroma de Dhat) e a sua abordagem em cuidados de saúde primários.

\section{DESCRIÇÃO DO CASO}

Descreve-se o caso de um utente do sexo masculino, 44 anos, natural do Bangladesh. É casado e pai de duas

*Médica Interna de MGF, USF da Baixa, ACeS Lisboa Central. filhas (11 e 7 anos), mas reside sozinho em Portugal desde 2010. Tem 12 anos de escolaridade e trabalha como secretário. Nega antecedentes pessoais e familiares relevantes, hábitos toxicofílicos ou toma de medicação habitual. Na análise do genograma e da psicofigura de Mitchell destaca-se a morte da mãe quando o utente tinha 15 anos, a boa relação com uma das irmãs (que aponta como pessoa de referência durante a adolescência) e a relação distante com elementos da família do sexo masculino (principalmente com o irmão mais velho).

Recorreu à Unidade de Saúde Familiar (USF) pela primeira vez em novembro de 2017, onde pediu consulta não programada por sensação de morte iminente - "Sinto que vou morrer a qualquer momento" (sic). Na sequência desse pedido foram realizadas três consultas: uma consulta não programada no próprio dia e 


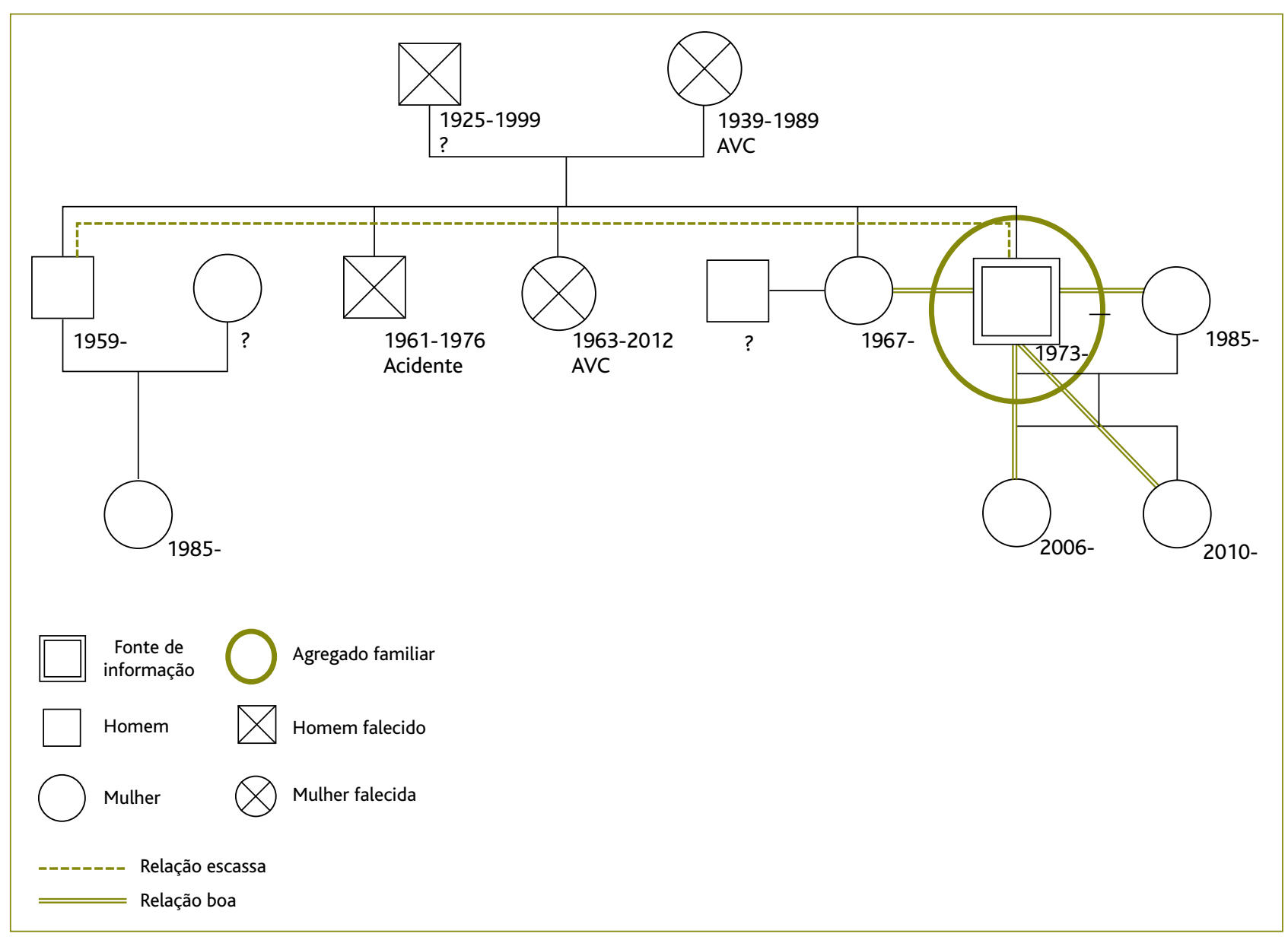

Figura 1. Genograma e psicofigura de Mitchell realizados a 28/11/2017.

duas consultas programadas nas quatro semanas seguintes.

Nas consultas, realizadas em inglês, o utente referiu episódios de tristeza, ansiedade, palpitações, dificuldade de concentração, cansaço e insónia terminal com vários meses de evolução. Dizia sentir-se sozinho - "sinto muita falta da minha família, principalmente da minha mulher" (sic)-, estar mais isolado - "só saio de casa para trabalhar e rezar" (sic) - e ter dificuldade em fazer amigos, "principalmente senhoras... não posso dizer a uma mulher que ela é bonita... eu sou casado..." (sic). Estes sintomas começaram a ter impacto na sua vida pessoal e profissional: "Em nove anos de trabalho nunca pedi férias. Se não posso ir ao Bangladesh, não preciso de férias. Mas agora senti-me tão mal que pedi férias de um dia para o outro!" (sic).
Afirmou que o quadro tinha iniciado depois da emissão de um "líquido branco" (sic) pelo pénis enquanto urinava, e por vezes ao acordar, e que a perda desse líquido fê-lo sentir-se fraco e era "por isso é que [estava] doente" (sic). Negou contactos sexuais recentes ou outros sintomas genito-urinários. Explicou que nunca tinha falado sobre este tema com um familiar, amigo ou profissional de saúde porque tinha medo que não compreendessem os seus receios.

Apresentava-se ansioso, choroso e verborreico. Não foram observadas alterações relevantes ao exame objetivo, incluindo ao exame dos órgãos genitais.

Durante as consultas foi realizada escuta ativa, com reconhecimento do sofrimento e tranquilização do utente. As consultas de reavaliação permitiram explorar as preocupações do utente, desmistififcar crenças 
TABELA 1. Queixas comuns na síndroma de Dhat

\begin{tabular}{|c|c|c|c|c|}
\hline Queixas somáticas & Queixas cognitivas & Queixas sexuais & Queixas de ansiedade & Queixas de depressão \\
\hline $\begin{array}{l}\text { Dores musculares } \\
\text { generalizadas } \\
\text { Sensação de mal-estar } \\
\text { geral } \\
\text { Fadiga } \\
\text { Falta de força } \\
\text { Dor abdominal } \\
\text { Dores articulares } \\
\text { Cefaleia } \\
\text { Sensação de perda de } \\
\text { peso } \\
\text { Perda de apetite } \\
\text { Obstipação } \\
\text { Disúria } \\
\text { Sensação de febre }\end{array}$ & $\begin{array}{l}\text { Perda de memória } \\
\text { Problemas de } \\
\text { concentração }\end{array}$ & $\begin{array}{l}\text { Disfunção eréctil } \\
\text { Impotência } \\
\text { Ejaculação precoce }\end{array}$ & $\begin{array}{l}\text { Preocupação excessiva } \\
\text { Dispneia } \\
\text { Palpitações } \\
\text { Tensão muscular }\end{array}$ & $\begin{array}{l}\text { Tristeza } \\
\text { Sensação de desespero } \\
\text { Anedonia } \\
\text { Irritabilidade } \\
\text { Sensação de culpa } \\
\text { Vergonha } \\
\text { Insónia ou sono } \\
\text { excessivo }\end{array}$ \\
\hline
\end{tabular}

Adaptação de: Prakash, Sharan e Sood (2018) ${ }^{1}$ e Deb e Balhara (2013). ${ }^{10}$

acerca da sua saúde e propor estratégias para combater o isolamento. O plano, elaborado de forma partilhada com o utente, incluía também a realização de hemograma, função renal, enzimas hepáticas, função tiroideia, urina II e análises para avaliação de doenças sexualmente transmissíveis (nomeadamenteVIH, sífilis e hepatite B).

Após as consultas descritas, o utente solicitou contacto telefónico. Explicou que tinha sido autorizado pelo Serviço de Estrangeiros e Fronteiras a viajar para fora do país e ia visitar o Bangladesh, sem saber a data do regresso, pelo que não poderia comparecer à consulta agendada. Estava animado e referia melhoria das queixas, principalmente as de ansiedade, assim como a resolução do quadro de insónia. O próprio expressou vontade de marcar consulta quando voltasse do Bangladesh. Foi-lhe facultado também o $e$-mail institucional da médica de família, para o qual enviou o resultado das análises de sangue e urina, que não tinham alterações.

\section{COMENTÁRIO}

Os diferentes aspetos culturais e sociais podem influenciar a forma como os conceitos de saúde e doença são compreendidos. ${ }^{8-9}$ Devido à crescente globaliza- ção, a multiculturalidade assume um papel cada vez mais relevante nas sociedades e o médico de família, como prestador de cuidados de primeira linha, pode contactar com utentes de vários países. Embora exista pouca informação acerca de síndromas culturais, o seu conhecimento poderá ser importante na abordagem de doentes que recorram à consulta com queixas inespecíficas.

A síndroma de Dhat encontra-se descrita no DSM-V (no capítulo «Conceitos culturais de sofrimento») e na CID-10 (no capítulo «Other specified neurotic disorders») e, embora seja mais frequentemente relatada no Sul da Ásia, foram também confirmados casos na Europa e no continente americano. Consiste na preocupação do utente em que a perda de sémen seja causadora de perda de energia vital e, consequentemente, de doença. ${ }^{1-7}$

Os pacientes com síndroma de Dhat recorrem à consulta com múltiplas queixas inespecíficas (Tabela 1) após a emissão de fluído interpretado como sémen durante episódios de ejaculação noturna, micção, defecação, masturbação ou excitação sexual. ${ }^{1-7}$ Apontam como possíveis razões de aparecimento da síndrome a visualização de filmes ou revistas pornográficas, ${ }^{1,4,6} \mathrm{O}$ excesso de desejo sexual, ${ }^{3-4,6}$ o estilo de vida pouco 
saudável (consumo excessivo de álcool, sedentarismo) ${ }^{3-4}$ ou em consequência de preocupações financeiras. ${ }^{1,4,6}$

É geralmente descrita em homens jovens (com média de idades entre os 22 e 26 anos), solteiros ou recém-casados e com níveis baixos de literacia acerca de saúde sexual. ${ }^{1,3-4,7} \mathrm{Um}$ estudo de $2018^{1}$ salienta que o aparecimento da síndrome pode ter relação com a falta de oportunidades para a prática de relações sexuais. Até ao momento não foi encontrada relação com crenças religiosas (sendo descrito em utentes hindus, muçulmanos e sikhs), status socioeconómico, escolaridade, atividade profissional ou habitação., ${ }^{1,-4,10}$

Alguns estudos apontam que esta síndroma possa ter base no sistema Ayurvédico, que admite que a saúde de um indivíduo depende do equilíbrio de sete componentes fundamentais do corpo (os sete dhatus), sendo o sémen (dhatu) o mais importante - a perda deste elemento é interpretada como perda de força vital. ${ }^{1,6-7,10}$ É importante, porém, ter em conta que a relação sexual entre casais não é considerada uma forma de perda de energia, já que existe «troca de dhatus» entre os parceiros. ${ }^{1}$

Como em muitos casos, esta síndroma é observada em utentes com diagnóstico de perturbação depressiva, perturbação de ansiedade,,$^{1,4,6-7,10}$ perturbação de sintomas somáticos ${ }^{1,10} \mathrm{e} / \mathrm{ou}$ abuso de substâncias, ${ }^{4}$ pelo que não é ainda possível concluir se a síndroma de Dhat é uma manifestação de uma destas patologias ou se se trata de uma entidade separada. ${ }^{2,4} \mathrm{Em} 2014$, Grover e colaboradores criaram o Comprehensive Questionnaire for Assessment of Dhat Syndrome, de forma a avaliar as crenças dos utentes sobre as causas, sintomas e possíveis tratamentos desta síndroma. ${ }^{5-6}$ Não existem outros instrumentos de avaliação disponíveis e existe pouca informação acerca dos possíveis tratamentos desta síndroma. ${ }^{10} \mathrm{O}$ consenso atual passa por uma abordagem individualizada e multidisciplinar, centrada no conceito de «humildade cultural». Este conceito define que o profissional de saúde deveria reconhecer os seus próprios vieses culturais, de forma a poder abordar da melhor forma utentes com culturas ou crenças diferentes da sua. ${ }^{8-9} \mathrm{Na}$ síndroma de Dhat, esta abordagem passa por escuta ativa, desmistificação de crenças e aumento da literacia em saúde mental e sexual..$^{1,5,7,10}$ Outros tratamentos propostos são a psicoterapia, ${ }^{1,7,10}$ a terapia cognitivo-comportamental ${ }^{7}$ e o uso de antidepressivos e/ou ansiolíticos. . $^{1,70}$
Neste artigo relata-se o caso de um indivíduo proveniente do Sul da Ásia, com baixo nível de informação acerca de saúde sexual, sem atividade sexual há vários anos, que recorreu à consulta com várias das queixas descritas na Tabela 1 e que associava o aparecimento dessas queixas à emissão de um fluído branco pelo pénis, interpretado como sémen. O conhecimento da existência de síndromas culturais, e desta síndroma em particular, permitiu uma abordagem individualizada, evitando medidas terapêuticas desadequadas. Realizou-se escuta terapêutica, tranquilização do utente e desmistificação de crenças, valorizando as suas queixas e respeitando o seu contexto cultural. Os contactos subsequentes demonstraram o estabelecimento de uma relação terapêutica entre médico e doente e assistiu-se a uma evolução favorável do caso.

A divulgação deste caso teve como objetivo a sensibilização dos médicos de família para a existência de síndromas culturais, a necessidade de abordagem individualizada nestes casos e a possibilidade de questionar o utente sobre os motivos pelos quais considera estar doente.

\section{AGRADECIMENTOS}

À Dra. Tatiana Consciência e ao Dr. Martino Gliozzi, pelo aconselhamento e sugestões.

\section{REFERÊNCIAS BIBLIOGRÁFICAS}

1. Prakash S, Sharan P, Sood M. A qualitative study on psychopathology of Dhat syndrome in men: implications for classification of disorders. Asian J Psychiatr. 2019;35:79-88.

2. Khambaty M, Parikh RM. Cultural aspects of anxiety disorders in India. Dialogues Clin Neurosci. 2017;19(2):117-26.

3. Grover S, Avasthi A, Gupta S, Dan A, Neogi R, Behere PB, et al. Phenomenology and beliefs of patients with Dhat syndrome: a nationwide multicentric study. Int J Soc Psychiatry. 2016;62(1):57-66.

4. Prakash S, Sharan P, Sood M. A study on phenomenology of Dhat syndrome in men in a general medical setting. Indian J Psychiatry. 2016;58(2):129-41.

5. Kar SK, Sarkar S. Dhat syndrome: evolution of concept, current understanding, and need of an integrated approach. J Hum Reprod Sci. 2015;8(3):130-4.

6. Grover S, Avasthi A, Aneja J, Shankar G, Mohan MR, Nehra R, et al. Comprehensive questionnaire for assessment of Dhat syndrome: development and use in patient population. J Sex Med. 2014;11(10):2485-95.

7. Udina M, Foulon H, Valdés M, Bhattacharyya S, Martín-Santos R. Dhat syndrome: a systematic review. Psychosomatics. 2013;54(3):212-8.

8. Prasad SJ, Nair P, Gadhvi K, Barai I, Danish HS, Philip AB. Cultural humility: treating the patient, not the illness. Med Educ Online. 2016;21:30908. 
9. Yeager KA, Bauer-Wu S. Cultural humility: essential foundation for clinical researchers. Appl Nurs Res. 2013;26(4):251-6.

10. Deb KS, Balhara YP. Dhat syndrome: a review of the world literature. Indian J Psychol Med. 2013;35(4):326-31.

\section{CONFLITO DE INTERESSES}

A autora declara não ter quaisquer conflitos de interesse.
ENDEREÇO PARA CORRESPONDÊNCIA

Sara Antunes

E-mail: sararl213@gmail.com

https://orcid.org/0000-0003-4145-217X

Recebido em 22-09-2019

Aceite para publicação em 27-07-2020

\section{ABSTRACT}

\section{CULTURE AND FAMILY MEDICINE: A DHAT'S SYNDROME CASE}

Introduction: Dhat's syndrome is the concern that the loss of semen causes loss of vital energy and, consequently, causes disease. It occurs mainly in young men from the south of Asia and presents in the form of nonspecific symptoms (such as weakness, sadness, and anxiety). This case intends to discuss the importance of a patient's socio-cultural context and its approach in a primary health care setting.

Case description: It is presented the case of a 44-year-old man, from Bangladesh, with no relevant personal and family history. A member of a nuclear family in his home country (in phase IV of Duvall's life cycle), he emigrated to Portugal in 2010, where he lives alone. With no previous follow-up at the Health Center (USF), he requested an unscheduled consultation with complaints of weakness, anxiety, and a feeling of imminent death. He associated the symptoms with the emission of white liquid during urination. He denied having sexual contacts in the last 7 years. Considering the possible diagnostic of Dhat syndrome, an active listening posture was adopted. Reassessment appointments were scheduled, to which the patient adhered, and during which the doctor-patient therapeutic relationship was established, with progressive improvement of the symptoms. Comment: Multiculturality plays an increasingly important role in societies. The family doctor, as a gatekeeper, can play an important role in identifying cultural syndromes. Knowledge of this particular syndrome allowed for an individualized approach, avoiding inappropriate therapeutic measures, with a favorable outcome of the case.

Keywords: Cultural diversity; Dhat syndrome; Family practice. 